\title{
EMI Shielding by Plating Technique and Its Performance on the Shielding Effectiveness
}

\author{
Tai, Min Fee, Kok, Swee Leong, Kenichiroh Mukai
}

\begin{abstract}
In the effort to miniaturize the electronic packaging, plating on mold compound has been in intensively studied as a solution to replace the metal can for Electro-Magnetic Interference (EMI) shielding. Plating technique is capable to reduce 5 to 10 times space compare to the metal can. $A$ pre-shielded package can be done by plating in an array form before send to the die cut. This will enable shielding in bulk hence reduce the manufacturing foot print and cost. In our past research work, we had demonstrated a feasible manufacturing process, and the plated product had reached the high reliable performance [1]. Following the research, we design a series of microstrip patch antennas to evaluate the effectiveness of EMI shielding by the plating technique, in frequency of $2.65,6.26$ and $10.0 \mathrm{GHz}$. Antennas are coated with conformal coat ink as the insulator, followed by plating of the nickel-phosphorous (NiP) and the pure copper $(\mathrm{Cu})$. The capability of plated metal shield to attenuate the antenna receiving power, is used to evaluate the performance of EMI shielding effectiveness (SE). Result showed a direct co-relation of the metal thickness towards EMI shielding effectiveness, but it come into saturation when we further increase the thicknesses. Besides, test also shown material selection on copper versus nickel-phosphorous with its high differences on conductivity and permeability properties, resulting in different EMI shielding performance at different frequency. The combination of 2 different metals enhance the EMI shielding result compare to single metal.
\end{abstract}

KEYWORDS: EMI shielding, shielding effectiveness, antenna, nickel plating, copper plating.

\section{INTRODUCTION}

The overcrowding of wireless signal in surrounding had led to EMI shielding becoming more important. According to industry news, Apple is applying EMI shield technology to variety of digital chips, radio frequency, connectivity (wireless LAN, Bluetooth) chips and others that also include application processor and modems. It is a measure to increase device's performance while decreasing electromagnetic interference to major chips and to solve people's fear on electromagnetic waves from smartphones [2].

When electronic devices receive strong electromagnetic waves, unwanted electric currents can be induced in the circuit and thus cause unintended operations or interference with the intended operations. If the energy

Revised Manuscript Received on October 22, 2019.

Tai, Min Fee, Faculty of Manufacturing Engineering, Universiti Teknikal Malaysia Melaka, Hang Tuah Jaya, 76100 Durian Tunggal, Melaka, Malaysia. MFS Technology (M) Sdn Bhd, Lot 5, Batu Berendam FTZ III, 75350, Melaka, Malaysia

Kok, Swee Leong, Faculty of Manufacturing Engineering, Universiti Teknikal Malaysia Melaka, Hang Tuah Jaya, 76100 Durian Tunggal, Melaka, Malaysia.

Kenichiroh Mukai, Atotech USA Inc. 369 Inverness Pkwy \#350, Englewood CO 80112

Author's Email: mftai@yahoo.com; sweeleong@utem.edu.my. applied from the outside is too powerful, electronic devices can be damaged. Even if the energy applied from the outside is small, if it is mixed with the radio waves used for broadcasting and communication, it can cause loss of reception, abnormal noise in sound, or disrupted video at places where the radio waves for broadcasting and communication are weak. Such interference caused by external electromagnetic waves is called electromagnetic noise interference, and the electromagnetic waves that cause interference are called electromagnetic noise. Noise can cause interference with various electronic devices. The source of noise also varies. Noise that does not cause any interference with particular appliances (e.g. washing machines and refrigerators) but can seriously affect other devices (e.g. AM radios). Therefore, there are rules to suppress the noise generated from electronic devices to a certain level and to make sure that electronic devices operate correctly under a certain noise level so that we can use electronic devices with security. These rules are called noise regulations. If an electronic device is considered a source of noise, the occurrence of noise is called emission of noise. In contrast, if an electronic device is considered a victim of noise, the tolerance to noise is called immunity (noise tolerance). Noise regulations specify the emission and immunity of electronic devices. [3]

\section{2.0. EMI SHIELDING FABRICATION TECHNIQUE}

Alternative methods of EMI shielding are actively researched nowadays in the electronic industry [4]. The reasons are to fulfil the change of the challenging demand such as multifunctioning in a single device, which all chips need to be electromagnetically contained so it will not interfere others or being interfered during the operation. To substitute the metal can, a conformal coated metal on package (mainly on the outer layer of the mold compound) can help to save space up to few hundred micrometre, which is significantly in the electronic world. Conformal techniques which are currently available are screen-print, metal spray, sputtering and plating. The traditional old technique of screen printing has limitation on higher density design due to it thickness is high and very poor coverage on side wall [5].

The ability to coat on 3 dimension structures needs a high throwing power capability. In thin film metal coating, plating and Physical Vapor Deposition (PVD) are the preferred selection due to its well thickness control in thin layer and uniform. 


\section{EMI Shielding by Plating Technique and Its Performance on the Shielding Effectiveness}

There is a problem about the layer formation by present technology uses Chemical vapor Deposition (CVD) or Physical Vapor Deposition (PVD, also known as sputtering) which needs high vacuum and with the equipment of high cost. In the case of sputtering, it is difficult to form a conformal film in a high aspect ratio structure. Improvements have so far been made by achieving required film thickness when depositing seed layers within high aspect ratio via and at via bottoms by using long-term process; but it in addition prolonged the process time, such measure increases cost and lowers productivity. On the other hand, CVD needs high temperature process which affects the device performance. Despite the advances that have recently been made to improve the process of thin film barrier deposition by atomic layer deposition (ALD), issues remain in the development of advanced techniques for seed layers. Plating have advantages among others on low cost, thin layer control, good throwing power on dead corner coverage. Meanwhile the drawbacks plating is the environmentally challenge and backside coating is needed to cover the not-to-plated area [6].

Figure 1 illustrates the conventional versus new approach on EMI shielding by plating. Traditional EMI shielding by metal can is done on the last step after chip assembly. This method takes extra cost and it consumes more height and space. Means while by using plating on array form, shielding can be done on high volume scale, and reduce the size of package. During design on experiment, we put cost effective and ease of industrial mass production as factor of consideration. Electroless $\mathrm{NiP}$ is chosen due to it fast deposition, low running cost, and its high wear and corrosion resistance, which it can stand as the final finishing layer of the product. Besides, copper is also a cheap and easy available material. Copper has high conductivity and give high skin depth while handling the high frequency electro-magnetic wave.

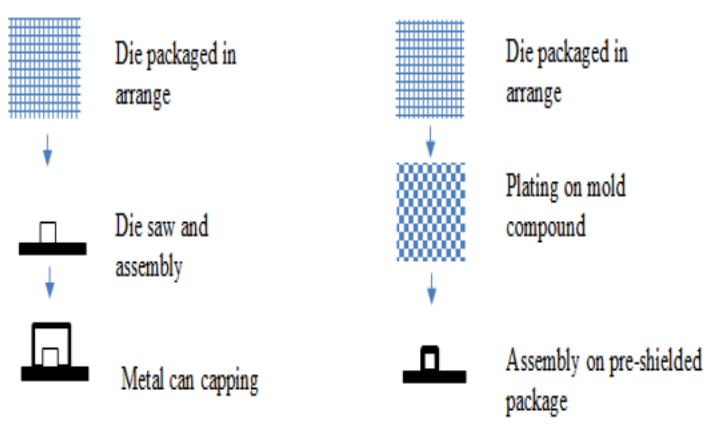

by metal can

a. New EMI Shielding by plating

Figure 1: Conventional vs New Approach of EMI shielding

\section{MEASURING OF EMI SHIELDING EFFECTIVENESS}

In summary, the shielding effectiveness in $\mathrm{dB}, S E_{d B}$ is,

$$
S E_{d B} \square \square R_{d B} \square \square A_{d B} \square \square M_{d B}
$$

where $R d B$ is the reflection loss, $A d B$ is the absorption loss, and $M d B$ is the multi-reflection loss. Equation is further explained by Figure 2. Due to the fact that the wave is attenuated to $1 / e$ at one skin depth, the multi-reflection loss can be ignored for the cases where the shielding thickness is much larger than the skin depth.

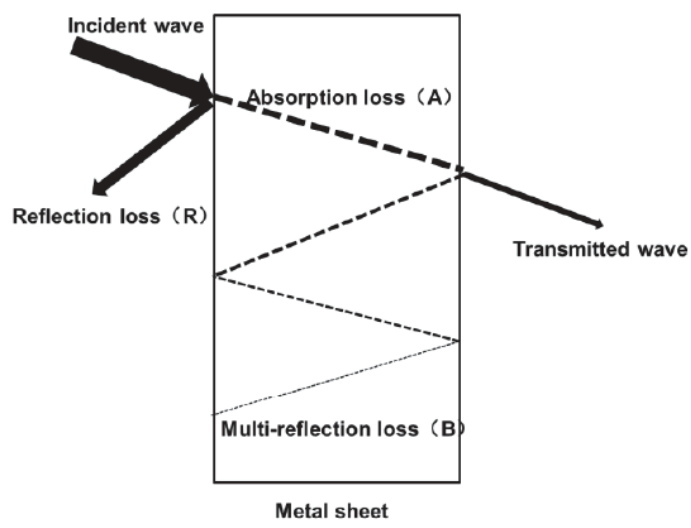

Figure 2: Electromagnetic Shielding Schematic Diagram [7]

The reflection loss is

$$
R=20 \log _{10}\left|\frac{\left(z_{0}+z_{1}\right)^{2}}{4 z_{0} z_{1}}\right|
$$

where $\mathrm{Z}_{0}$ represents the wave impedance of air and $\mathrm{Z}_{1}$ represents the wave impedance of the metal sheet. As

$$
Z_{1} \approx \sqrt{\frac{\mu_{1} \omega}{\sigma_{1}}}(1+j) \ll Z_{0}=120 \pi \omega
$$

$\mu_{1}$ and $\sigma_{1}$ are the relative permeability and conductivity of the metal sheet, respectively, formula (3) can be estimated as

$$
R=20 \log _{10}\left|\frac{z_{0}}{4 z_{1}}\right|=20 \log _{10}\left|\frac{30 \pi}{1+j}\right| \sqrt{\frac{\sigma_{1}}{\mu_{1} \omega}}
$$

The reflection loss is directly proportional to the conductivity of the metal sheet but inversely proportional to the relative permeability of the metal sheet.

The absorption loss is

$$
A=20 \log _{10} e^{\frac{\varepsilon}{8}}
$$

where $\mathrm{L}$ is the thickness of the metal sheet and $\delta$ is the skin depth.

b. Conventional EMI shieldifig $\overline{\sqrt{\frac{2}{\mu_{1} \omega \sigma_{1}}}}$

Formula (5) can be estimated as

$$
A=20 \log _{10} e^{\sqrt{\frac{\mathbb{R}_{1} \omega \sigma_{1}}{2}}}
$$

The absorption loss is directly proportional to the thickness, conductivity and relative permeability of the metal sheet. The multi-reflection loss is 


$$
\mathrm{B}=20 \log _{10}\left|1-\frac{\left(z_{0}-z_{1}\right)^{2}}{\left(z_{0}+z_{1}\right)^{2}} e^{-2(1+j) \frac{5}{8}}\right|
$$

As $\mathrm{Z}>>\mathrm{Z1}$, formula (8) can be estimated as

$$
\mathrm{B}=20 \log _{10}\left|1-e^{-2(1+j) \frac{5}{8}}\right|
$$

If the thickness of the metal sheet is much larger than skin depth, incident wave will be attenuated significantly so that the multi-reflection loss will be negligible.

The EM shielding capability of a shield is escribed as shielding effectiveness, $S E$, which is defined as the ratio of the electric fields and magnetic field at a point without and with the shield as equation $10 \mathrm{a}$ and $10 \mathrm{~b}$,

$$
\begin{gathered}
\mathrm{SE}=20 \log _{10} \frac{E_{1}}{E_{0}} \\
\mathrm{SE}=20 \log _{10} \frac{\mathbb{B}_{1}}{H_{0}}
\end{gathered}
$$

where $E_{l}, H_{1}$ is the electrical and magnetic field at an arbitrary point without the shield, and $E_{2}, H_{2}$ is the electrical and magnetic field at the same point with the shield [7].

As power is quadratic of electrical and magnetic force, the equation can be further elaborated into

$$
\begin{gathered}
\mathrm{SE}=10 \log _{10}\left(\frac{P_{1}}{P_{2}}\right), \text { for power unit in watt } \\
S_{E}=10 \log _{10} P_{1}-10 \log _{10} P_{2} \\
S_{E}(\text { in } d B)=P_{1}-P_{2}
\end{gathered}
$$

for power unit in $\mathrm{dBm}$

From table 1, we notice different material will have various physical characteristic on conductivity and permeability, which causing selection of material is important to determine the shielding effective of design.

Table 1: Electrical conductivity relative to copper $\left(\sigma_{\mathrm{r}}\right)$ and relative magnectic permeability $\left(\mu_{\mathrm{r}}\right)$ of selected material. [8]

\begin{tabular}{lcccc}
\hline Material & $\sigma_{r}$ & $\mu$ & $\sigma_{r} \mu_{f}$ & $\sigma / \mu^{\prime}$ \\
\hline Silver & 1.05 & 1 & 1.05 & 1.05 \\
Copper & 1 & 1 & 1 & 1 \\
Gold & 0.7 & 1 & 0.7 & 0.7 \\
Aluminum & 0.61 & 1 & 0.61 & 0.61 \\
Brass & 0.26 & 1 & 0.26 & 0.26 \\
Bronze & 0.18 & 1 & 0.18 & 0.18 \\
Tin & 0.15 & 1 & 0.15 & 0.15 \\
Lead & 0.08 & 1 & 0.08 & 0.08 \\
Nickel & 0.2 & 100 & 20 & $2 \times 10^{-3}$ \\
Stainless steel (430) & 0.02 & 500 & 10 & $4 \times 10^{-5}$ \\
Mumetal & & & & \\
$\quad$ (at 1 kHz) & 0.03 & 20,000 & 600 & $1.5 \times 10^{-6}$ \\
Superpermalloy & & & & \\
$\quad$ (at 1 kHz) & 0.03 & 100,000 & 3,000 & $3 \times 10^{-7}$ \\
\hline
\end{tabular}

In the electroless $\mathrm{NiP}$ plating, by increasing of Phosphorous $(\mathrm{P})$ content is a common method to improve the corrosion resistivity, but the electrical resistivity will also increase to double on after $11 \% \mathrm{P}$ (in weigh \%) versus the pure nickel.

\section{Effect of EN deposit composition on electrical resistivity}

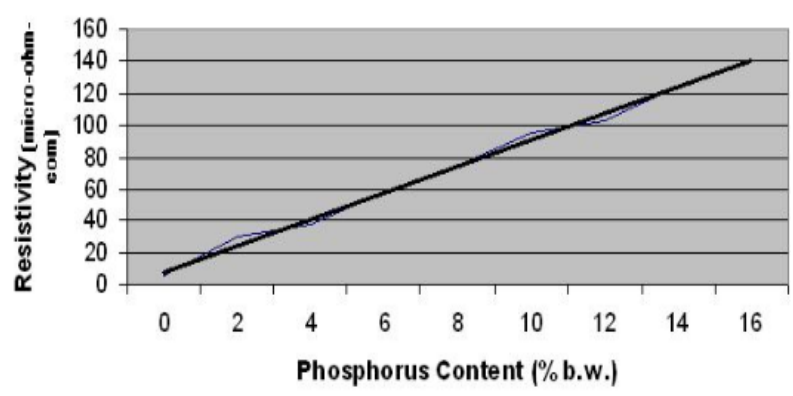

Figure 3: Effect of Resistivity vs Phosphorous content

\section{EXPERIMENTAL SET-UP}

3 microstrip patch antennas are designed to test the reacceptance of signal at $2.75 \mathrm{GHz}, 6.26 \mathrm{GHz}$ and $10.0 \mathrm{GHz}$ as shown in Figure 4. Selection of frequency are meet to current $4 \mathrm{G}$ bandwidth, and extend to higher frequency for future $5 \mathrm{G}$ expansion need. The antennas are fabricated on FR4 substrate with permittivity and thickness of 5.4 and 1.6 $\mathrm{mm}$ respectively. The substrate has copper thickness of 0.035 $\mathrm{mm}$ at both sides. In this design, quarter-wave matching approach is used to get bigger bandwidth. The antenna is fed with $50 \Omega$ SMA using the edge feeding technique.

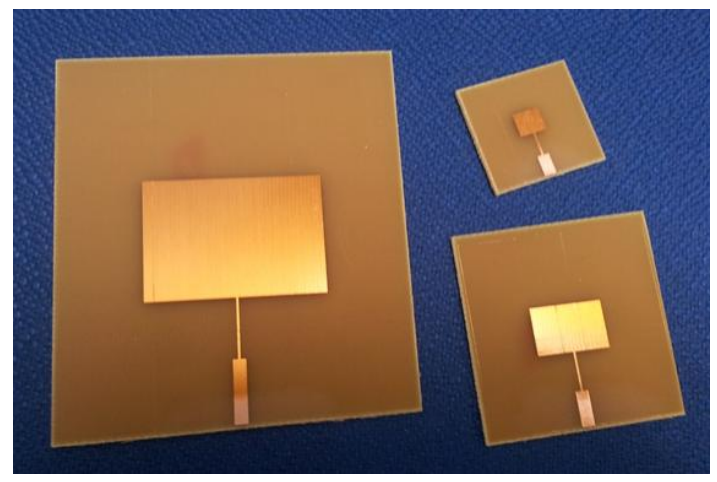

Figure 4: Fabrication of Antenna

Sample fabrication begins with copper clad laminating with FR4 dielectric. Antenna pattern is formed by printing of etch resist on copper clad; the unwanted $\mathrm{Cu}$ is removed with cupric chloride etchant. When the pattern is formed, the etch resist is stripped and exposed the pattern in $\mathrm{Cu}$. The fabrication process is then followed by using conformal coating ink to cover the antenna, to act as an insulator to antenna layer. A series of antenna samples are dried for 2 days to ensure the ink are fully dried and cured. After that the samples are ready for the metal plating process.

As conformal coating ink is not conductive, therefore surface activation is needed; this is begun with palladium colloid to form a seed layer prior to plating. This is done by the catalytic metallic nuclei of metal Palladium on the non-catalytic surface; by generating an electrochemical oxidation-reduction reaction, 


$$
\mathrm{Pd}^{2+}+\text { reducing agent } \rightarrow \mathrm{Pd}^{0}+\text { oxidized agent }
$$

where $\mathrm{Pd}^{2+}$ is the metallic ion and $\mathrm{Pd}^{0}$ is the metal catalyst. In many case the preferred reducing agent is $\mathrm{Sn}^{2+}$, which would be oxidized into $\mathrm{Sn}^{4+}$.

The fabrication process is followed by electroless plating of nickel-phosphorous (NiP). NiP is a preferred process due to it low cost and the throwing power is essentially perfect. Metallization of $\mathrm{NiP}$ is an autocatalytic deposition of ion $\mathrm{Ni}^{2+}$ reduced by sodium hypophosphate [9].

$\mathrm{Ni}^{2+}+2 \mathrm{e}$ (reducing agent) $\rightarrow \mathrm{NiP}+\mathrm{By}$-product

Table 2: Bath Components for NiP Electroless Plating [9]

\begin{tabular}{|c|c|}
\hline $\mathrm{Chemical}$ & Concentration \\
\hline $\mathrm{NiCl}_{2} \cdot 6 \mathrm{H}_{2} \mathrm{O}$ & $40-50 \mathrm{~g} / \mathrm{l}$ \\
\hline $\mathrm{NaH}_{2} \mathrm{PO}_{2} \cdot \mathrm{H}_{2} \mathrm{O}$ & $30-40 \mathrm{~g} / \mathrm{l}$ \\
\hline $\mathrm{NH}_{4} \mathrm{Cl}$ & $60-80 \mathrm{~g} / \mathrm{l}$ \\
\hline $\mathrm{C}_{6} \mathrm{H}_{8} \mathrm{O}_{7} \cdot \mathrm{H}_{2} \mathrm{O}$ & $40-50 \mathrm{~g} / \mathrm{l}$ \\
\hline $\mathrm{Na}_{2} \mathrm{~B}_{4} \mathrm{O}_{7} \cdot 10 \mathrm{H}_{2} \mathrm{O}$ & $10-20 \mathrm{~g} / \mathrm{l}$ \\
\hline
\end{tabular}

After plating a layer of $\mathrm{NiP}$, the test is then carry on with additional deposition of copper $(\mathrm{Cu})$ with thicknesses 3,5, and 10um on top of conductive layer 20um NiP. As electroless $\mathrm{Cu}$ deposition rate is very slow and costly, process selection switch to electrolytic $\mathrm{Cu}$. The sample is connected in a $\mathrm{Cu}$ plating bath where the sample itself is acting as the cathode (negative charge), and another $\mathrm{Cu}$ bar as the anode (positive source) and the electrolyte is made up of acidic copper sulphate solution. The setup of the experiment is shown in Figure 5, when the rectifier is turned on, copper starts to deposit from anode to cathode.

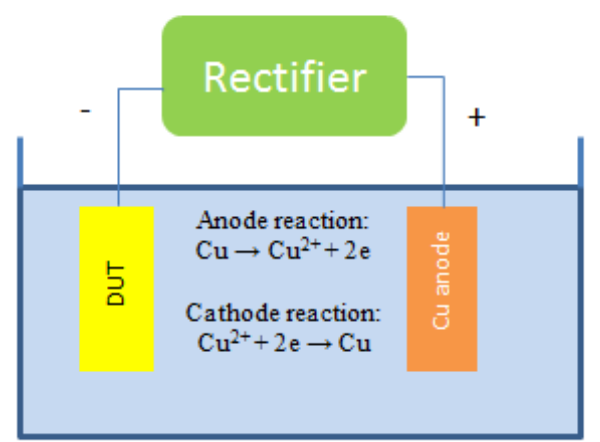

Figure 5: Electrolytic Plating on Copper

Table 3: Bath Component for Acid Copper Solutions [10]

\begin{tabular}{|c|c|}
\hline Chemical reagents & Concentration \\
\hline $\begin{array}{c}\text { Copper sulphate, } \\
\mathrm{CuSO}_{4} .5 \mathrm{H}_{2} \mathrm{O}\end{array}$ & $200-250 \mathrm{~g} / \mathrm{l}$ \\
\hline Sulfuric acid, $\mathrm{H}_{2} \mathrm{SO}_{4}$ & $60-100 \mathrm{~g} / 1$ \\
\hline Chloride & $40-80 \mathrm{mg} / 1$ \\
\hline Leveller & $20-30 \mathrm{~g} / 1$ \\
\hline Brightener & $0.5-1.5 \mathrm{~g} / 1$ \\
\hline
\end{tabular}


Repeat test with additional electrolytic copper plating

Figure 6: Process Flow of DUT Preparation

The measuring of EMI shielding effectiveness is based on the attenuation of antenna after shielded by metal. Devices under test (DUT) are place on connection to signal analyser, facing to horn of signal generator, with distance of $50 \mathrm{~cm}$. The signal generator is tune to $2.75 \mathrm{~Hz}, 6.26 \mathrm{~Hz}$, and $10.0 \mathrm{~Hz}$ to match the acceptance signal of antenna; with power of $20 \mathrm{dBm}$. The powers received by antenna are recorded by signal analyser, and data is used for SE calculation (Figure 7).

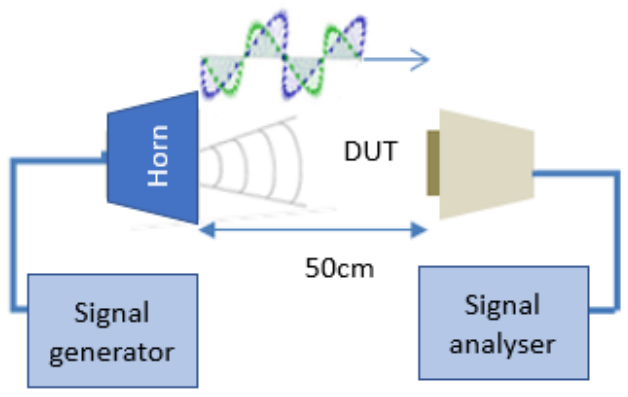

\section{Figure 7: Measuring of Electromagnetic Shielding Effective}

\section{EXPERIMENT RESULTS \& DISCUSSION} shielding layer to absorb and to reflex the electromagnetic wave from the horn transmitter. The minimum power receiving from the antenna is indicating the better EMI shielding performance.

Design of experiment starts with microstrip patch antennas, the NiP plated samples with thickness of 1, 3, 5, 10, 20, 30 are tested at 2.75, 6.26 and 10.0 GHz. Antennas are placed as DUT shown in Figure 7, facing oppositely to a horn which impose noise with various of frequency, in fixed energy 20 $\mathrm{dBm}$. The signal analyzer which connected to the DUT will measure the energy received after attenuated by the plated metal. The differences of energy received between metal-shielded and non-metal-shielded is used to study the EMI shielding efficiency (Figure 8).

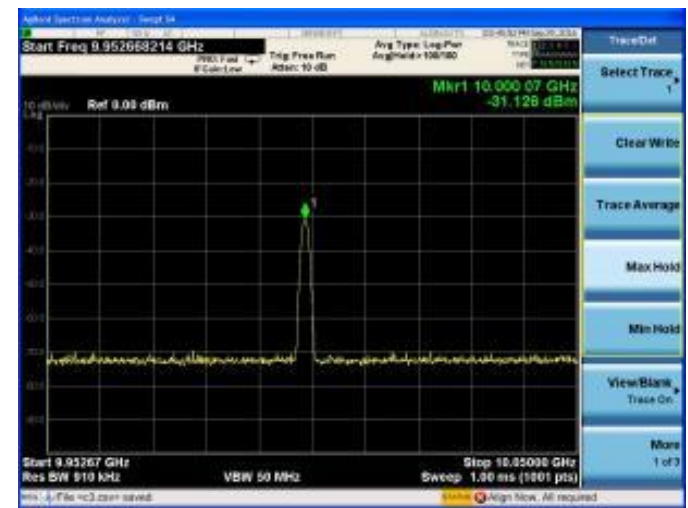

Figure 8: Raw Data on Measuring EMI Shielding Efficiency by Signal Analyzer improved over the increase of NiP thickness, but its reach the limitation after 10-20um thicknesses. For frequency of
In principle, the plated metal $(\mathrm{NiP}$ or $\mathrm{Cu})$ will act as the

From the first result in Figure 9, we see the SE is steadily

$2.75 \mathrm{~Hz}$, a SE of above $17 \mathrm{~dB}$ is achievable with more than 20um of NiP; mean whiles, for $6.25 \mathrm{GHz}$, maximum $\mathrm{SE}$ of $10 \mathrm{~dB}$ is recorded after $20 \mathrm{um}$. It is also found that lower frequency signal $(2.75 \mathrm{GHz})$ is easier to shield compare to the higher frequency $(6.26,10 \mathrm{GHz})$ by NiP material. In noise frequency of $10 \mathrm{GHz}$, the shielding efficiency show only around $3 \mathrm{~dB}$ even $20-30 \mathrm{um}$ of NiP is applied.

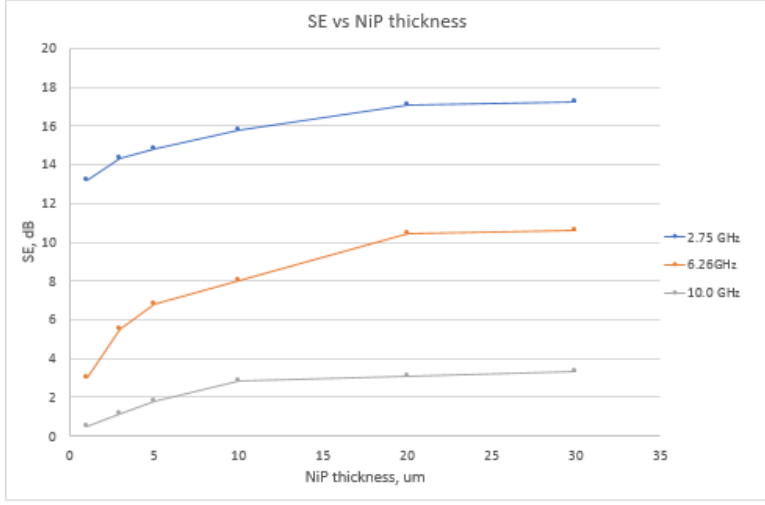

Figure 9: Shielding Effectiveness vs Nickel-Phosphorous Thickness

From the Equation 5, the absorption loss is

$$
A=20 \log _{10} e^{\frac{\varepsilon}{8}}
$$

where $\mathrm{L}$ is the thickness of the metal sheet. When the thickness of NiP increase (L increase), the absorption loss towards external noise will increase, hence higher metal thickness gives better SE until it is limited by others factor such as skin effect.

In order to extend the shielding effectiveness, a different material, Copper $(\mathrm{Cu})$ is plated on top of 20um NiP surface.

The NiP 20um and Cu plated samples with thickness of 1, 5, 10 are tested at 2.75, 6.26 and $10.0 \mathrm{GHz}$.

On the additional $\mathrm{Cu}$ plated on top of $20 \mathrm{um} \mathrm{NiP}$, we see $\mathrm{Cu}$ is capable improving the SE. On $2.75 \mathrm{GHz}$, the SE increase to $20 \mathrm{~dB}$, whereby the $6,26 \mathrm{GHz}$ increase into $14 \mathrm{~dB}$, and $10 \mathrm{GHz}$ increase to $9 \mathrm{~dB}$. In general, increasing the $\mathrm{Cu}$ thickness after 5um shows its limitation to further improve the SE.

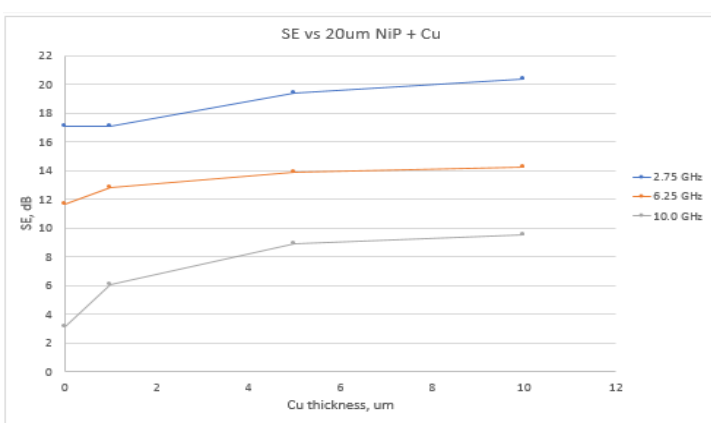

Figure 10: Further Improvement of SE with additional Cu plated on top of $20 \mathrm{um} \mathrm{NiP.}$

From the Figure 10 test result, we also notice $\mathrm{Cu}$ give better SE capability vs NiP. $\mathrm{Cu}$ has better relative conductivity 1.0, which is 5 times than pure Ni (Table 1). Increasing the phosphorus content will further increases the electrical resistivity of the film. 
$\mathrm{NiP}$ at around $8 \%$ of Phosphorous will have 10 times lower conductivity than pure $\mathrm{Ni}$ (Figure 3.) From the Equation 7 and 8, the reflection loss and absorption lost is proportional to the conductivity. $\mathrm{Cu}$ with higher conductivity gives better reflection loss and absorption loss capability, which indicate its higher EMI shielding capability.

Additional $\mathrm{Cu}$ in high frequency $10.0 \mathrm{GHz}$ show SE increase from $3 \mathrm{~dB}$ into $9 \mathrm{~dB}$, which is almost $200 \%$ of the improvement. Electromagnetic radiation from the high frequency penetrates only at the near surface region of conductor layer, which normally called skin effect. Most materials have the relative permeability $\mu_{\mathrm{r}}$ at near to 1 , including copper. The exceptions are nickel, iron, etc, which are called ferro-magnetic materials. Metals such as nickel can have permeability as high as 100 or more.

From equation 6 , the skin depth, $\delta$, note it is dominated by the conductivity, $\mu$ and permeability, $\sigma$. For copper, when relative conductivity, $\mu_{\mathrm{r}}=1$, and $\sigma_{\mathrm{r}}=1, \delta_{\mathrm{r}}=44.7 \times 10^{-6}$ at 10.0 $\mathrm{GHz}$; and for nickel, when relative conductivity, $\mu_{\mathrm{r}}=0.2$, and $\sigma_{\mathrm{r}}=100, \delta_{\mathrm{r}}=3.16 \times 10^{-6}$ at $10.0 \mathrm{GHz}$. Ni is 5 times lower conductivity than $\mathrm{Cu}$, but the ultimate conductivity of a nickel thin film can be as low 7\% compare to $\mathrm{Cu}$. This is because the skin depths are much thinner due to the relative permeability term, which is often reported at 100 for nickel. Permeability is a strong function of frequency once we reach few $\mathrm{GHz}$. The magnetic domains can't follow the fast-changing magnetic field anymore and relative permeability drops down towards unity. Permeability and frequency are both in the denominator of the skin-depth formula [11].

Nevertheless, NiP is still a prefer layer in combination to $\mathrm{Cu}$ in designing of plating solution of EMI shielding. NiP with its ferromagnetic characteristic, the shielding at very lower frequency (lower than $10 \mathrm{MHz}$ ) could be significant. Besides, NiP with its good corrosion resistance and high hardness can protect the $\mathrm{Cu}$ layer from oxidation and the mechanical damage. Such combination compensates the weaknesses on each material and form the perfect match.

\section{CONCLUSION}

With the combination with plated $\mathrm{NiP}$ and $\mathrm{Cu}$, a range of 9-20 dB shielding effectiveness can be achieved. $\mathrm{NiP}$ to combined with $\mathrm{Cu}$ is a better EMI shield compared to NiP alone. SE does not increase with metal thickness. Saturation of performance found at thickness of 20um NiP and $5 \mathrm{um} \mathrm{Cu}$, beyond this it come to flat. Selection of thickness and material will depend on the EMI shielding design requirement and the target cost. 3 of the EM frequencies have shown same pattern of changes although the $\mathrm{SE}$ is better at lower frequency. The conformal plating on mold compound provides alternative solution for EMI shielding in IC packaging.

\section{REFERENCES}

1. MF Tai et al, Adhesion Enhancement for Electroless Plating on Mold Compound for EMI Shielding with Industrial Test Compliance, IEEE-ICSE2014 Proc. Kuala Lumpur,2014

2. Patently Apple, Retrieved from http://www.patentlyapple.com/patently-apple/2016/02/apple-is-addi ng-emi-shielding-technology-to-the-iphone-7.html, February 16 , 2016.

3. https://www.murata.com/en-eu/products/emc/emifil/ knowhow/basic/ chapter01-pl.
4. L.G. Bhatgadde S. Joseph, Electroless Techniques For EMI Shieldings, Advanced Centre For Research In Electronics, Indian Institute Of Technology, Powai, Mumbai, 1997. pp. 2

5. Jimmy-Dinh V Hoang et. al, Breakthorugh Packaging Level Shielding Technique and EMI Effectiveness Modeling and Chracterization, IEEE $66^{\text {th }}$ Electronic Components and Technology Conference 2016 , Skyworks Solution, California. pp1-3

6. F. Inoue et.al, Conformal Deposition of Electroless Barrier and Seed Layers in TSV with $\mathrm{Au}$ Nano Particle Catalyst, Department of Mechanical Engineering, Kansai University. pp 1-2

7. Fankun Jiang, Ming Li, Liming Gao, Research on conformal EMI shielding Cu/Ni layers on package, 15th International Conference on Electronic Packaging Technology, 2014

8. DDL Chung, Material for Electromagnetic Interference Shielding, ASM International, 5 Jan2000.

9. Mordechay Schlesinger, Electroless Deposition of Nickel, Modern Electroplating, pp. 447-458, 2010.

10. Jack W. Dini And Dexter D. Snyder, Electrodeposition of Copper, Modern Electroplating, pp 33-78, 2010

11. Microwaves101. Retrieved from

a. https://www.microwaves101.com/encyclopedias/high-permeability-m aterials

12. Dong-Lin Zhao *, Xia Li, Zeng-Min Shen, Microwave absorbing property and complex permittivity and permeability of epoxy composites containing $\mathrm{Ni}$-coated and Ag filled carbon nanotubes, Composites Science and Technology 68, pp 2902-2908, 2008.

13. Liao Kuo-Hsien, Alex, Chi-Hong, Chan, Shen Chia Hsien, Lin, I-Chia; Huang Hsin Wen, Novel EMI Shielding Methodology on Highly Integration SiP Module, CPMT Symposium Japan, 2nd IEEE, 2012.

14. Nozad Karim, Jingkun Mao, Jun Fan, Improving Electromagnetic Compatibility Performance of Packages and SiP Modules Using a Conformal Shielding Solution, Asia-Pacific International Symposium on Electromagnetic Compatibility, Beijing, China, April 12 - 16, 2010

15. Atotech Data Sheet, Neoganth MV Activator (V), Doc.-No. 32-4800-08, used date 07.12.2009

16. Atotech Data Sheet, Aurotech CNN mod., used date 17.10.2012

17. Atotech Data Sheet, Printoganth $H(H)$, used date 09.11.2016

18. Atotech Data Sheet, Cupracid HL, used date 09.11.2004.

19. Peters Data Sheet, Conformal Coat s1_0100000e_009. 